\title{
Dysregulation of miR-23b-5p promotes cell proliferation via targeting FOXM1 in hepatocellular carcinoma
}

\author{
Xinchen Yang ${ }^{1}$, Shikun Yang ${ }^{1}$, Jinhua Song ${ }^{1}$, Wenjie Yang $\mathbb{E}^{1}$, Yang $\mathrm{Ji}^{1}$, Feng Zhang ${ }^{1}$ and Jianhua Rao ${ }^{1}$
}

\begin{abstract}
Growing evidence demonstrates that MicroRNAs (miRNAs) play an essential role in contributing to tumor development and progression. However, the underlying role and mechanisms of miR-23b-5p in hepatocellular carcinoma (HCC) formation remain unclear. Our study showed that miR-23b-5p was downregulated in the HCC tissues and cell lines, and lower expression of miR-23b-5p was associated with more severe tumor size and poorer survival. Gain- or loss-of-function assays demonstrated that miR-23b-5p induced G0/G1 cell cycle arrest and inhibited cell proliferation both in vitro and in vivo. GRT-PCR, western blot and luciferase assays verified that Mammalian transcription factor Forkhead Box M1 (FOXM1), upregulated in HCC specimens, was negatively correlated with miR23b-5p expression and acted as a direct downstream target of miR-23b-5p. In addition, miR-23b-5p could regulate cyclin D1 and c-MYC expression by directly targeting FOXM1. Further study revealed that restoration of FOXM1 neutralized the cell cycle arrest and cell proliferation inhibition caused by miR-23b-5p. Taken together, our findings suggest that miR-23b-5p acted as a tumor suppressor role in HCC progression by targeting FOXM1 and may serve as a potential novel biomarker for HCC diagnosis and prognosis.
\end{abstract}

\section{Introduction}

Hepatocellular carcinoma (HCC) is a common malignancy and the third leading cause of cancer-related mortality worldwide, particularly in China with high prevalence of hepatitis B virus (HBV)/hepatitis C virus $(\mathrm{HCV})$ infection $^{1,2}$. In recent years, despite the great progression in HCC treatment, the 5-year survival rate of patients with HCCs remains unsatisfactory due to the high frequency of recurrence and metastasis ${ }^{3,4}$. Given the asymptomatic nature in the early stages, most HCC patients are diagnosed at advanced stages. Therefore, it is critical to elucidate the mechanisms underlying the HCC

\footnotetext{
Correspondence: Feng Zhang (zhangfeng1958@hotmail.com) or Jianhua Rao (raojh@njmu.edu.cn)

${ }^{1}$ Hepatobiliary Center, The First Affiliated Hospital of Nanjing Medical University; Key Laboratory of Liver Transplantation, Chinese Academy of Medical Sciences, NHC Key Laboratory of Liver Transplantation, Nanjing, China These authors contributed equally: Xinchen Yang, Shikun Yang, Jinhua Song Edited by Alessandro Rufini
}

progression and explore the effective therapeutic strategies to improve the diagnosis and treatment of $\mathrm{HCC}^{5}$.

MicroRNAs are endogenous, single-stranded, small non-coding RNAs regularly 21 to 24 nucleotides in length. By binding to the $3^{\prime}$-untranslated region ( $\left.3^{\prime} \mathrm{UTR}\right)$ of the target messenger RNA (mRNA), they could modulate gene expression at post-transcriptionally level and affect multiple essential biological processes, such as cell proliferation, movement, metastasis, differentiation, apoptosis and death ${ }^{6-8}$. Previous researches have shown the role of miR-23b-5p in a variety of pathological and physiological processes. Oumarou et al. ${ }^{7}$ found that miR-23b-5p was involved in promotion of cardiac hypertrophy and dysfunction via activating HMGB2 signaling pathway. You et al. ${ }^{8}$ confirmed the role of miR-23b-5p in thermogenic program by regulating brown adipogenesis. In addition, increasing number of researches have illustrated the suppressive function of miR-23b-5p in the progression of malignant tumors ${ }^{9,10}$. However, the specific biological

\section{(c) The Author(s) 2021}

(c) Open Access This article is licensed under a Creative Commons Attribution 4.0 International License, which permits use, sharing, adaptation, distribution and reproduction cc) in any medium or format, as long as you give appropriate credit to the original author(s) and the source, provide a link to the Creative Commons license, and indicate if changes were made. The images or other third party material in this article are included in the article's Creative Commons license, unless indicated otherwise in a credit line to the material. If material is not included in the article's Creative Commons license and your intended use is not permitted by statutory regulation or exceeds the permitted use, you will need to obtain permission directly from the copyright holder. To view a copy of this license, visit http://creativecommons.org/licenses/by/4.0/. 
function and mechanisms of miR-23b-5p in HCC has yet to be determined.

Mammalian transcription factor Forkhead Box M1 (FOXM1) belongs to the extensive family of Forkhead transcription factors, characterized by a conserved protein domain called Forkhead or winged-helix domain, which known as a key regulator in tumor growth and cell-cycle regulation $^{11}$. FOXM1 could exert oncogenic functions by regulating multiple downstream targets in human malignant tumors ${ }^{12,13}$, such as CCND1 and $\mathrm{c}-\mathrm{MYC}^{14,15}$. Increasing studies have shown that FOXM1 is extensively overexpressed in most human malignancies, including HCC, breast cancer and lung cancer, and its increased expression may serve as a biomarker and indicate the poor outcome of patients ${ }^{16-19}$. Meanwhile, recent investigation suggested that miRNAs could be involved in the functions of FOXM1 ${ }^{20,21}$. However, little is known about the functional link between miR-23b-5p and FOXM1 in HCC.

In this study, we verified the downregulated miR-23b-5p expression in HCC tissues and cell lines, and found that HCC patients with higher miR-23b-5p expression showed a better overall survival. Furthermore, CCK-8 assay, EdU assay and colony formation assays demonstrated that overexpressed miR-23b-5p could inhibit cell proliferation. Mechanistically, miR-23b-5p could induce HCC cell cycle arrest at the G1 phase by directly binding to the 3'UTR of FOXM1. These findings indicated that miR-23b-5p functioned as a tumor suppressor miRNA in the progression of liver cancer by decreasing the expression of FOXM1, and miR-23b-5p may serve as a novel diagnostic and prognostic biomarker for HCC.

\section{Results}

miR-23b-5p expression is downregulated in HCC tissues and cell lines

To identify whether miR-23b-5p was dysregulated in HCC tissues, we investigated miR-23b-5p expression in 60-paired HCC tissues and adjacent peritumor tissues by qRT-PCR. We observed lower miR-23b-5p expression level in tumor tissues compared with adjacent nontumor tissues (Fig. 1A). Consistent with this result, the analysis of TCGA database also showed downregulated level of miR-23b-5p in HCC tissues (Supplementary Fig. S1A). In addition, compared to immortalized human hepatocyte LO2 cells, miR-23b-5p expression in HCC cells (Hep3B, HepG2, HCCLM3, SMMC-7721, Huh-7, MHCC-97H and MHCC-97L) was significantly decreased (Fig. 1B). Moreover, we empolyed four paired $\mathrm{HCC}$ and peritumoral tissues for FISH analysis of miR-23b-5p and further confirmed the lower miR-23b-5p expression in HCC tissues (Fig. 1C). To investigate the clinicopathological features of miR-23b-5p in HCC, patients involved in this study were divided into high or low-group based on median value. As shown in Table 1, lower miR-23b-5p expression was significantly associated with larger tumor size $(P<0.05)$. In addition, we performed Kaplan-Meier analysis on these $60 \mathrm{HCC}$ patients, and revealed that lower miR-23b-5p expression was correlated with poorer overall survival (Fig. 1D, $P<0.01$ ).

\section{miR-23b-5p inhibits HCC cell growth and induces cell cycle arrest}

As shown in Fig. 1B, the HCC cell line HCC-LM3 and Hep3B exhibited the lowest and highest expression level of miR-23b-5p respectively. We transfected pre-miR-23b$5 p$ into HCC-LM3 cells and transfected miR-23b-5p inhibitor into Hep3B cells respectively. qRT-PCR was performed to confirm the transfection efficiency. HCCLM3 cells treated with pre-miR-23b-5p presented a marked increase in the miR-23b-5p expression level, while Hep3B cells transfected with miR-23b-5p inhibitor notably inhibited the miR-23b-5p expression level compared to negative control group (Fig. 1E). To evaluate the function of miR-23b-5p in cell proliferation, CCK-8 assays, EdU assays and colony formation assays were conducted. In the CCK8 assays, compared with the control groups, we found that upregulation of miR-23b-5p significantly inhibited the proliferation of HCC-LM3 cells, whereas downregulation of miR-23b-5p significantly promoted the growth of Hep3B cells (Fig. 2A). Meanwhile, ectopic expression of miR-23b-5p suppressed the colony formation ability of HCC-LM3 cells. In contrast, knockdown of miR-23b-5p promoted colony formation in Hep3B cells (Fig. 2B). Consistent with these results, EdU assays showed that stable overexpression of miR-23b-5p decreased the numbers of EdU-positive nuclei of HCCLM3 cells compared with the controls, while stable knockdown of miR-23b-5p increased the numbers of EdU-positive nuclei (Fig. 2C). As miR-23b-5p notably inhibited HCC cell proliferation, we aimed to ascertain whether miR-23b-5p could regulate the cell cycle of HCC cells. As determined by flow cytometry, the effects of miR23b-5p dysregulation on the cell cycle were showed in Fig. $2 \mathrm{D}$. We observed that overexpression of miR-23b-5p in the HCC-LM3 cells presented an obvious increase in G0/ G1 phase, while knockdown of miR-23b-5p in the Hep3B cells led to a marked decrease in G0/G1 phase compared with the control group. These data demonstrated that overexpressed miR-23b-5p inhibited HCC cell growth and induced G0/G1 cell cycle arrest.

FOXM1 is upregulated in human HCC tissues and cell lines and correlated with poor survive

As miR-23b-5p induced cell proliferation inhibition, we utilized bioinformatic databases (TargetScan and miRWalk) to predict the potential downstream target of miR23b-5p. Among them we particularly interested in 

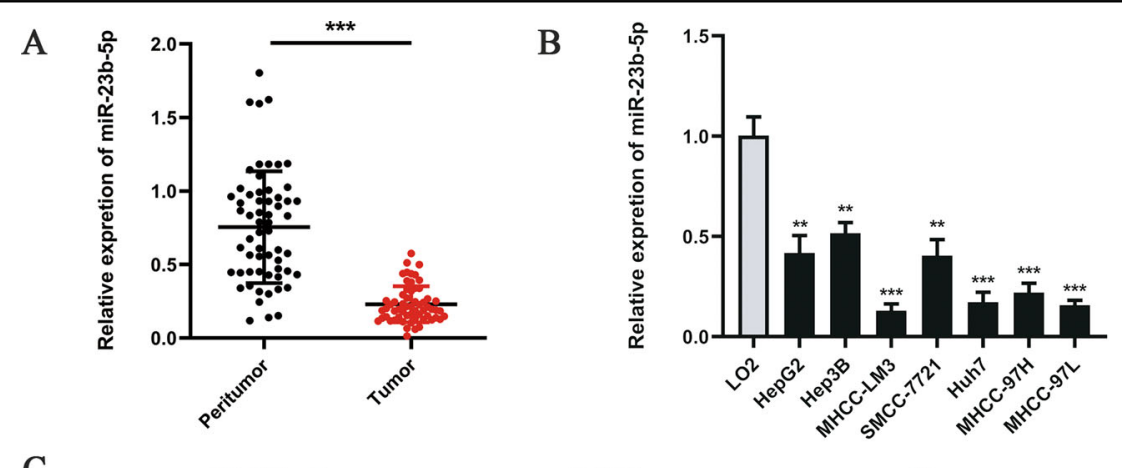

C

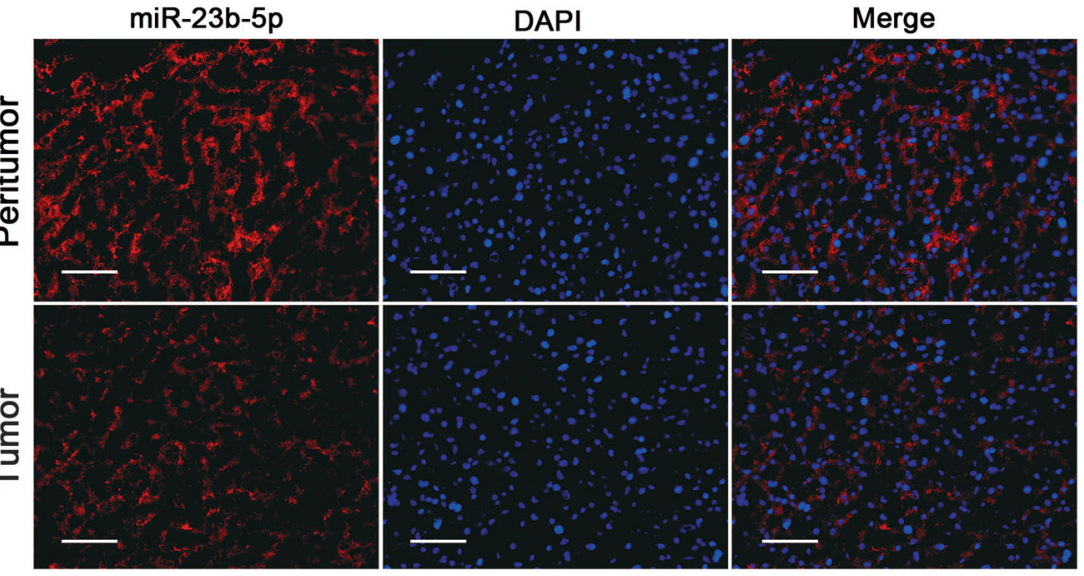

D

$\mathrm{E}$

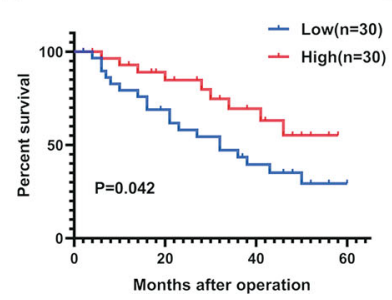

HCC-LM3
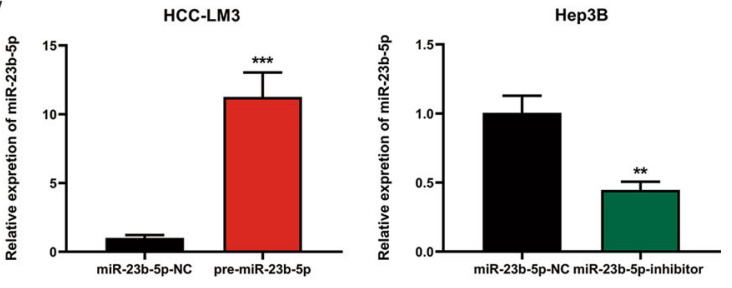

Fig. 1 miR-23b-5p is downregulated in HCC tissues and cell lines. A The mRNA levels of miR-23b-5p in 60-paired human HCC and adjacent normal tissues were tested by qRT PCR. B The mRNA levels of miR-23b-5p expression in HCC cell lines and normal LO2 cells. C Expression of miR-23b$5 p$ in HCC and adjacent normal tissues was measured by FISH assay (scale bars, $100 \mu \mathrm{m}$ ). D Kaplan-Meier analysis for overall survival of 60 patients with HCC resection according to the miR-23b-5p expression. E The HCC-LM3 and Hep3B cells were transfected with lentivirus overexpressing miR23b-5p (pre-miR-23b-5p) or lentivirus with short hairpin RNA targeting miR-23b-5p (miR-23b-5p-inhibitor). Cells transfected with empty lentiviral vectors served as a negative control (NC). The miR-23b-5p expression levels were analyzed by qRT-PCR. All data are presented as the mean \pm S.E.M. $\left({ }^{*} P<0.05,{ }^{* *} P<0.01\right.$, and $\left.{ }^{* *} P<0.001\right)$.

FOXM1, since FOXM1 had been reported to play a central role in the control of cell proliferation and was widely recognized as a key player in cell cycle progres$\operatorname{sion}^{16,18}$. Previous studies revealed that FOXM1 was highly expressed in HCC tissues and closely associated with poor prognosis ${ }^{22-24}$. We analyzed FOXM1 expression from TCGA database (Supplementary Fig. S1B) and in 60-paired HCC tissues and adjacent normal tissues by qRT-PCR (Fig. 3A). Compared to adjacent normal tissues, FOXM1 expression was significantly lower in HCC tissues and this result was further verified by immunohistochemistry (Fig. 3D). Consistent with the above data,
mRNA level of FOXM1 was also lower in HCC cells compared to LO2 cell (Fig. 3B). In addition, Kaplan-Meier analysis indicated that higher FOXM1 expression was correlated with a remarkable shorter overall survival time (Fig. 3C).

\section{miR-23b-5p directly targets FOXM1 in HCC cells}

We have already determined the miR-23b-5p and FOXM1 expression in different HCC cell lines via qRTPCR (Figs. 1B and 3B). The results showed that miR-23b$5 \mathrm{p}$ was expressed at a low level, while FOXM1 expression levels were increased compared to those observed in LO2 
Table 1 Association between miR-23b-5p expression and clinicopathologic features of patients with hepatocellular carcinoma.

\begin{tabular}{|c|c|c|c|c|}
\hline \multirow[b]{2}{*}{ Characteristics } & \multirow[b]{2}{*}{ Number } & \multicolumn{2}{|c|}{ miR-23b-5p } & \multirow[b]{2}{*}{$P$-value } \\
\hline & & Low & High & \\
\hline All cases & 60 & 30 & 30 & \\
\hline Age(years) & & & & 0.592 \\
\hline$\leq 60$ & 22 & 12 & 10 & \\
\hline$>60$ & 38 & 18 & 20 & \\
\hline Gender & & & & 0.584 \\
\hline Female & 20 & 11 & 9 & \\
\hline Male & 40 & 19 & 21 & \\
\hline Liver cirrhosis & & & & 0.766 \\
\hline No & 15 & 7 & 8 & \\
\hline Yes & 45 & 23 & 22 & \\
\hline HBsAg status & & & & 0.573 \\
\hline Negative & 18 & 8 & 10 & \\
\hline Positive & 42 & 22 & 20 & \\
\hline a-fetoprotein(ng/ml) & & & & 0.197 \\
\hline$\leq 20$ & 31 & 13 & 18 & \\
\hline$>20$ & 29 & 17 & 12 & \\
\hline Tumor size(cm) & & & & $0.038^{\mathrm{a}}$ \\
\hline$\leq 5$ & 28 & 10 & 18 & \\
\hline$>5$ & 32 & 20 & 12 & \\
\hline Tumor multiplicity & & & & 0.795 \\
\hline Single & 33 & 17 & 16 & \\
\hline Multiple & 27 & 13 & 14 & \\
\hline Edmondson grade & & & & 0.195 \\
\hline$|-| \mid$ & 33 & 19 & 14 & \\
\hline III-IV & 27 & 11 & 16 & \\
\hline Tumor-node-metastasis stage & & & & 0.197 \\
\hline$|-| \mid$ & 29 & 17 & 12 & \\
\hline III-IV & 31 & 13 & 18 & \\
\hline
\end{tabular}

${ }^{\mathrm{a}}$ Indicates $P<0.05$.

cells. In addition, bioinformatic analysis of TCGA database also showed downregulated miR-23b-5p and upregulated FOXM1 level in HCC (Supplementary Fig. S1A, B). Furthermore, we compared FOXM1 and miR-23b-5p expression level in 60 pairs of HCC tissues and found that they were negatively correlated (Fig. 3E). Western blot and qRT-PCR demonstrated that upregulation of miR23b-5p resulted in decreased FOXM1 transcription and protein levels, while downregulated miR-23b-5p led to the opposite results (Fig. 3F-G). Luciferase reporter assay was used to further confirm that FOXM1 was a direct target of miR-23b-5p. Wild-type (WT) or mutant (MUT) FOXM1-3' UTR were inserted into a luciferase reporter vector. The results demonstrated that miR-23b-5p suppressed luciferase activity of the WT-FOXM1-3' UTR, whereas the luciferase activity of the MUT-FOXM1-3' UTR was almost unchanged (Fig. 3H, I). These data suggested miR-23b-5p could directly and negatively regulate FOXM1.

\section{Restoration of FOXM1 reverses the effects of miR-23b-5p on HCC cells}

To further illustrate that miR-23b-5p inhibited the proliferation of HCC cells by regulating FOXM1. HCCLM3 cells were transfected with pre-miR-23b-5p lentivirus. After $72 \mathrm{~h}$, the cells were transfected with LVFOXM1. FOXM1 upregulation was corroborated by qRTPCR and western blot (Fig. 4A, B). Through CCK-8, EdU and colony formation assays, we confirmed that FOXM1 restoration reversed the proliferation-inhibiting effect of miR-23b-5p (Fig. 4C-E). Moreover, flow cytometry analysis was conducted to investigate whether miR-23b-5p affected the cell cycle through the modulation of FOXM1 in HCC cells. It turned out that high expression of FOXM1 could abolish cell cycle arrest caused by upregulated miR-23b-5p in the HCC-LM3 cells (Fig. 4F). These findings indicated that restoration of FOXM1 could neutralize the influence of miR-23b-5p on HCC cell lines.

miR-23b-5p regulated CCND1/c-MYC by targeting FOXM1

In the preceding studies, we showed that miR-23b-5p could affect the cell cycle of HCC cancer cells. Therefore, we examined miR-23b-5p contribution to cell cycle by flow cytometry, and the results showed that miR-23b-5p could increase the ratio of cells in G0/G1 period and miR23b-5p inhibitor had the opposite effect (Fig. 2D). In addition, restoration of FOXM1 could neutralize the influence of miR-23b-5p. As we all know, cyclin D1 and cmyc were crucial for the regulation of cell proliferation and the cell cycle and were important targets of FOXM1 $1^{14,15}$. We have confirmed that miR-23b-5p could directly target FOXM1. Thus, we wondered whether miR23b-5p could regulate the expression levels of CCND1 and $\mathrm{c}-\mathrm{MYC}$ via FOXM1. We evaluated the expression levels of CCND1 and c-MYC by qRT-PCR and western blot after transfection with pre-miR-23b-5p and miR-23b$5 \mathrm{p}$-inhibitor lentivirus. HCC-LM3 cells overexpressing miR-23b-5p demonstrated a clear downregulation of CCND1 and c-MYC mRNA and protein expression level. Meanwhile, Hep3B cells with downregulated expression of miR-23b-5p showed a moderate increase in cyclin D1 and c-myc protein expression and marked upregulated mRNA expression level compared to the negative control 


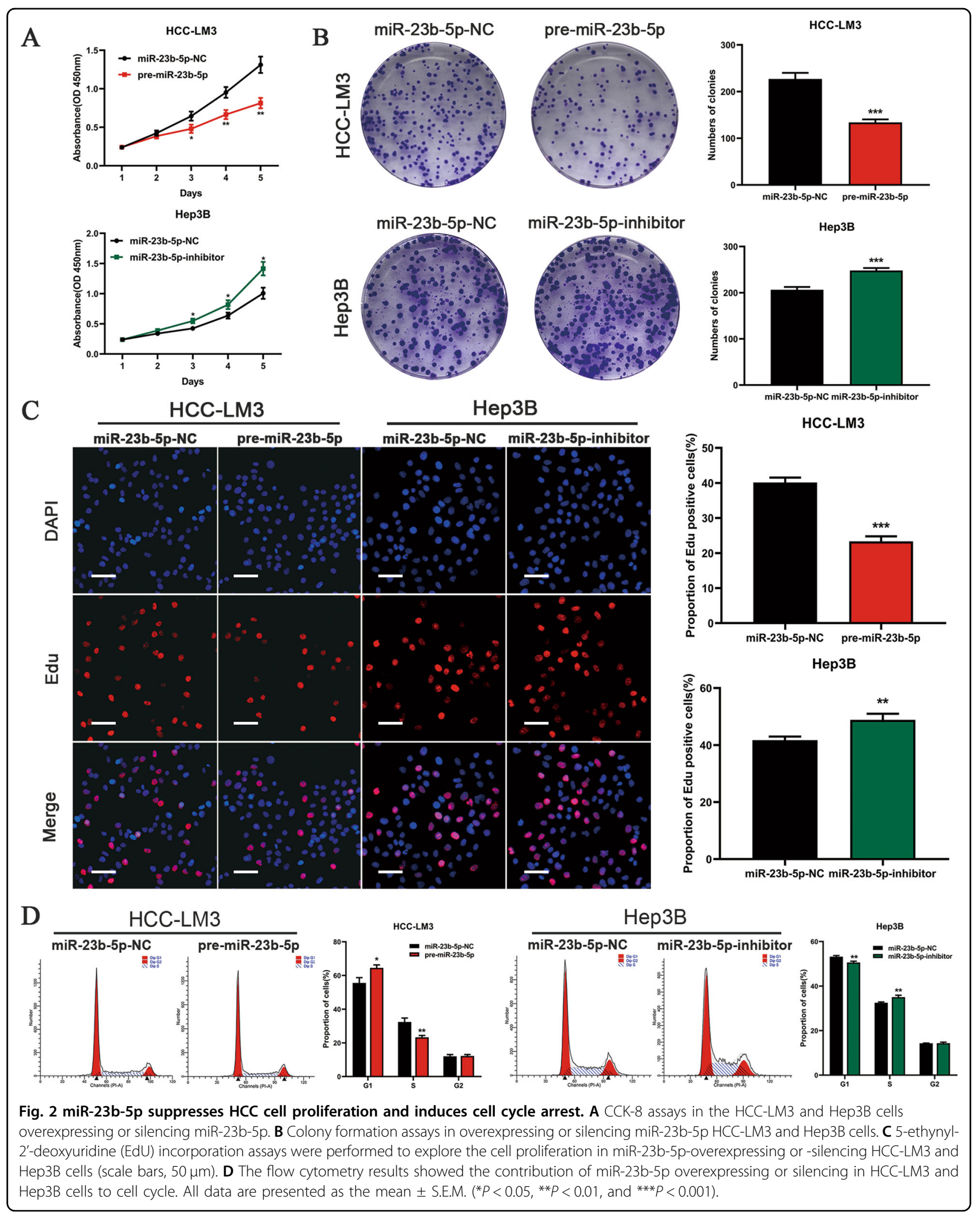




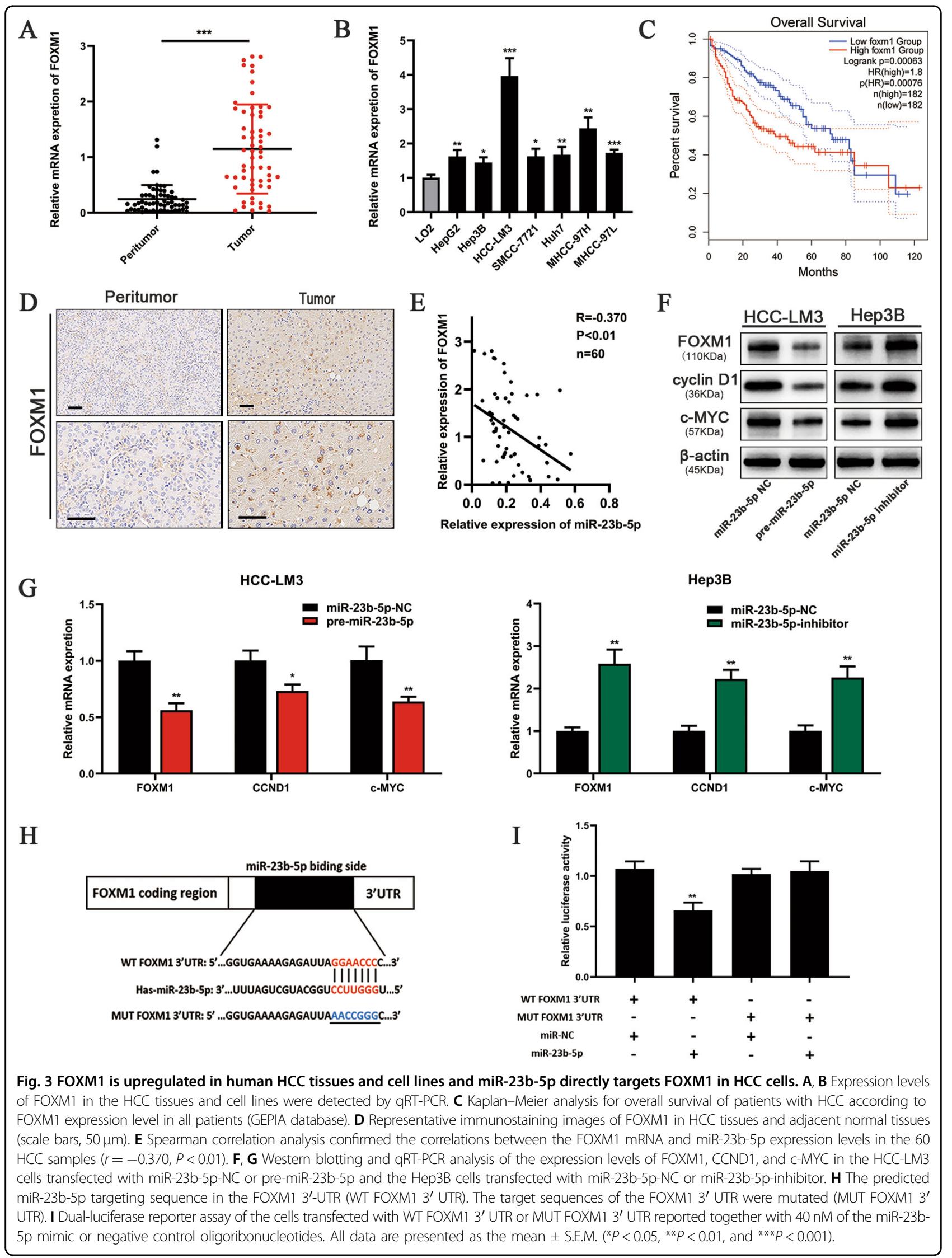




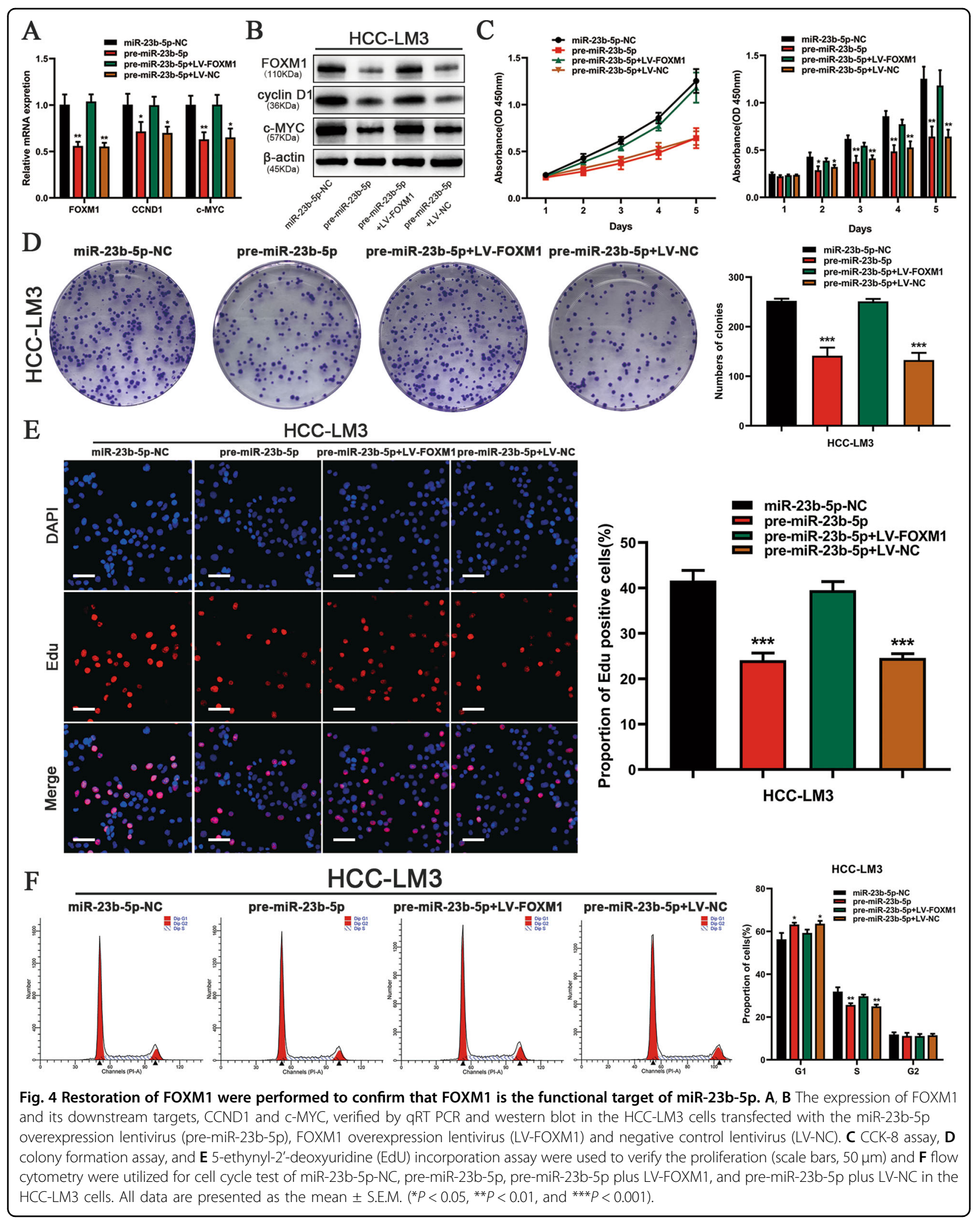



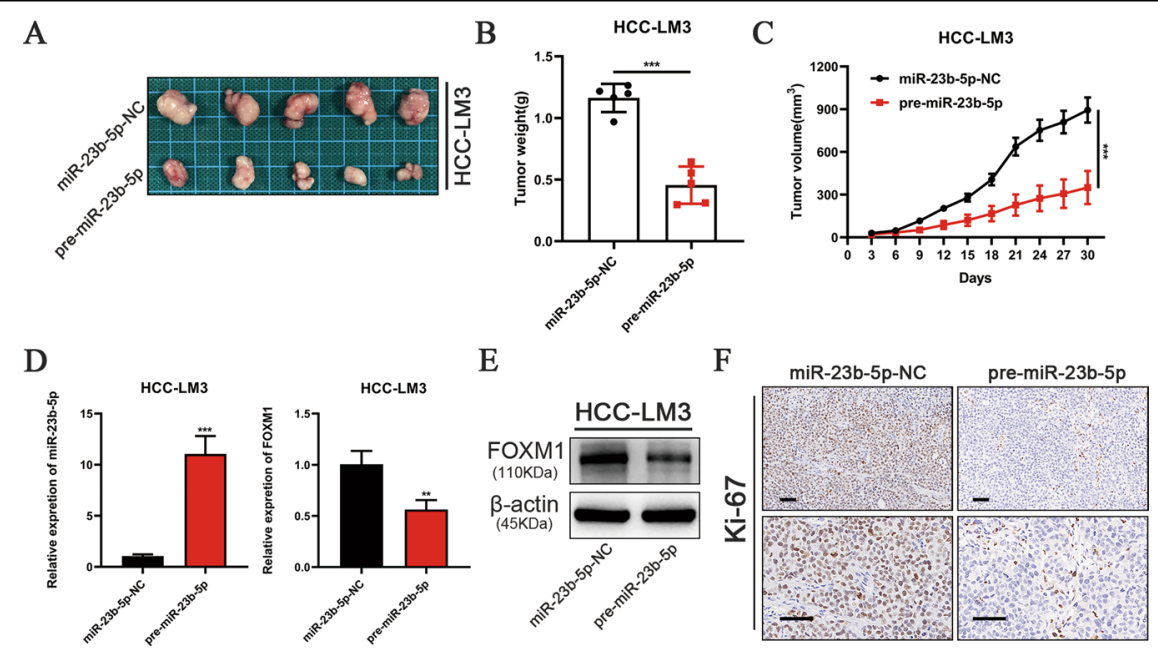

Fig. 5 miR-23b-5p suppresses xenograft tumor growth in vivo. A-C Photographs of tumors derived from the different groups of nude mice transfected with HCC-LM3-miR-23b-5p-NC and HCC-LM3-pre-miR-23b-5p cells. The tumors were measured for volume and average weight. D The miR-23b-5p and FOXM1expression levels of xenografts were analyzed by qRT-PCR. E The FOXM1 expression levels of xenografts were detected by western blot. F Ki67 staining of tumor tissues mice injected with HCC-LM3-miR-23b-5p-NC and HCC-LM3-pre-miR-23b-5p cells (scale bars, 50 $\mu$ m). All data are presented as the mean \pm S.E.M. $\left({ }^{*} P<0.05,{ }^{*} P<0.01\right.$, and $\left.{ }^{* *} P<0.001\right)$.

(Fig. 3F, G). Furthermore, we performed rescue experiments to verify the effects of restoration of FOXM1. As Fig. 4A, B indicated, decreased expression levels of CCND1 and c-MYC were restored when transfected with LV-FOXM1. These findings demonstrated that miR-23b$5 \mathrm{p}$ could regulate CCND1/c-MYC expression levels by targeting FOXM1.

\section{miR-23b-5p inhibits xenograft tumor formation}

To further investigate whether miR-23b-5p inhibited proliferation of HCC cells in vivo, HCC-LM3 cells transfected with miR-23b-5p-NC and pre-miR-23b-5p lentivirus were injected into nude mice. During tumor formation, the volume of tumor was recorded every 3 days, and the mice were euthanized 30 days later. The tumors generated from the HCC-LM3-pre-miR-23b-5p group were marked smaller and weight less than the NC group (Fig. 5A-C). Moreover, the results of qRT-PCR and western blot revealed that FOXM1 expression was significantly downregulated in the pre-miR-23b-5p group compared to the control group both in mRNA and protein level (Fig. 5D, E). In addition, immunohistochemistry showed that miR-23b-5p overexpression decreased the number of Ki67-positive cells (Fig. 5F). These results verified that miR-23b-5p suppressed tumor growth of $\mathrm{HCC}$ in vivo.

\section{Discussion and conclusion}

Numerous studies have confirmed that aberrantly expressed miRNAs could affect the proliferation and metastasis of HCC cells. In recent years, therapies based on miRNAs have been used in several preclinical models, including liver cancer ${ }^{25}$. Thus, the researches on miRNAs played important roles in providing new strategies for HCC diagnosis, prognosis, and therapy. Therefore, it is necessary to illuminate the relevant mechanisms between miRNAs and HCC.

Recently, the functional role of miR-23b-5p in malignant tumors has been proposed. Hu et al. ${ }^{9}$ found that miR-23b-5p was downregulated and acted as a sponge target of lncRNA to enhance the progression of lung adenocarcinoma. Farina et al. ${ }^{26}$ showed miR-23b-5p could influence prostate tumorigenesis and a bioinformatic analysis from Shan et al. ${ }^{10}$ revealed that miR-23b-5p may play an important role in the progression of hepatocellular carcinoma. Meanwhile, Warnecke-Eberz et al. ${ }^{27}$ isolated the exosomes from serum of patients with adenocarcinoma of the esophagus and showed that miR-23b-5p was merely detected in exosomes. Consistent with this result, Barrera-Ramirez et al. ${ }^{28}$ detected the exosomes from marrow-derived mesenchymal stromal cells in patients with acute myeloid leukemia and found that miR-23b-5p had a downregulated expression level in exosome. These data suggested that miR-23b-5p played an essential role in the progression of carcinoma formation. Nevertheless, the exact biological functions and molecular mechanisms of miR-23b-5p underlying HCC formation remain unknown. In this study, we demonstrated that the expression level of miR-23b-5p in HCC tissues and cell lines are downregulated. According to the data from $60 \mathrm{HCC}$ patients, we found that patients with low expression level of miR23b-5p had a larger tumor size and poorer outcomes compared to those with high miR-23b-5p. Investigation in vivo and in vitro both confirmed the function of miR- 
23b-5p in cell proliferation and cell cycle distribution. We found that upregulation of miR-23b-5p could suppress cell growth and induced G0/G1 cell cycle arrest, whereas knockdown of miR-23b-5p showed opposite results, it made a great increase in proliferation and downregulated the population of cells in G0/G1 phase of the cell cycle. Taken together, our results suggested that miR-23b-5p functioned as a tumor suppressor with whose downregulation the progression of $\mathrm{HCC}$ could be promoted.

It is well known that miRNAs could typically bind and destabilize target mRNAs to exert its functions in regulating gene expression. Thus, we selected the target gene of miR-23b-5p with the help of biological tools and we focused on FOXM1 because of its strong biological function. FOXM1 was recognized as a potent oncogene accountable for poor prognosis in various carcinomas and acted a pivotal role in regulating DNA damage response, oxidative stress, drug resistance and progression of tumor growth $^{29-33}$. FOXM1 was widely verified as a key player in cell cycle progression and could regulate a network of proliferation associated genes that are involved in mitotic spindle assembly, G1/S and G2/M transition and chromosome segregation ${ }^{16,17,34-36}$. In this study, we demonstrated that FOXM1 expression was increased and negatively correlated with miR-23b-5p expression in HCC tissues. Then, to validate whether miR-23b-5p could directly bind to the 3' UTRs of FOXM1, the luciferase activity assay was conducted. Furthermore, restoration experiments of FOXM1 was performed, we found that upregulated FOXM1 partially reversed the miR-23b-5p induced proliferation-inhibiting effect and cell cycle arrest in HCC cells. These data showed that miR-23b-5p could suppress HCC cell proliferation and induced G0/G1 cell cycle arrest via FOXM1.

To further investigate the mechanism of miR-23b-5p impacting on cell proliferation, we put our eyes on the network of proliferation associated genes regulated by FOXM1. Wierstra et al. ${ }^{15}$ reported that FOXM1 could transactivate the c-myc promoter by binding directly to its TATA-boxes and Wang et al. ${ }^{14}$ showed that FoxM1 also activated transcription of cyclin D1 promoter. Meanwhile, FOXM1 was involved in several significant signaling pathways such as the Wnt/ $\beta$-catenin pathway and interacts with its critical members, thereby contributing to signal transduction ${ }^{37}$. Zhang et al. ${ }^{38}$ verified that FOXM1 could interact with $\beta$-catenin, and promote $\beta$-catenin nuclear localization. When the FOXM1- $\beta$-catenin complex enters the nucleus, it further forms a transcriptional complex with T-cell factor (TCF) or lymphoid enhancer factor (LEF) to activate the expression of Wnt target genes including c-MYC and Cyclin D1 ${ }^{38}$. The c-MYC was well known as an oncogene contributes to the genesis of many human cancers ${ }^{39}$. Cyclin D1,belongs to the G1 cyclin family and plays a role in regulating the transition through the G1 phase of the cell cycle ${ }^{40}$. Accumulating studies have reported that Cyclin D1 functioned as an oncogene and its overexpression was associated with a variety of human malignancies, including $\mathrm{HCC}^{41,42}$. Thus, We hypothesized that cyclin D1 and c-myc may play an important role in miR-23b-5p impacting on HCC proliferation. In our study, we found the expression level of Cyclin D1 and c-MYC were decreased when miR-23b-5p was upregulated in HCC cells, whereas the downregulated miR-23b-5p group showed the opposite trend by western blot and qRT-PCR. Furthermore, we confirmed that when rescued the FOXM1 expression level in cells the changes caused by dysregulation of miR-23b-5p could be partially reversed.

In conclusion, our findings demonstrated that the expression levels of miR-23b-5p were downregulated in HCC tissues, which was related to poor prognosis of HCC patients. Overexpression of miR-23b-5p significantly reduced HCC proliferation and resulted in G0/G1 cell cycle arrest by targeting FOXM1. Therefore, miR-23b-5p may serve as a potential biomarker for the diagnosis and prognosis of $\mathrm{HCC}$, and provide a targeted approach to HCC therapy.

\section{Materials and methods}

\section{Tissue samples and cell lines}

In this study, 60-paired HCC and adjacent normal tissues from patients who underwent curative hepatectomy at The First Affiliated Hospital of Nanjing Medical University, China were tested. We obtained consent from the patients or their relatives before the collection of specimens. The study was approved by the Ethics Committee of Nanjing Medical University. All human HCC cells lines (Hep3B, HepG2, HCC-LM3, SMMC-7721, Huh-7, MHCC-97H and MHCC-97L) and the human normal liver cell line (LO2) were purchased from the Chinese Academy of Sciences (Shanghai, China). All cells were cultured in DMEM medium (Gibco, NY, USA) supplemented with $10 \%$ fetal bovine serum (Gibco), $100 \mathrm{U} / \mathrm{ml}$ penicillin (Gibco), and $100 \mathrm{mg} / \mathrm{ml}$ streptomycin (Gibco) at $37^{\circ} \mathrm{C}$ in a humidified cell incubator in a $5 \% \mathrm{CO}_{2}$ atmosphere.

\section{RNA isolation and quantitative real-time PCR (qRT-PCR) analysis}

We extracted total RNA from HCC tissues and cells using TRIzol reagent (Invitrogen, USA) following the manufacturer's instructions. Complementary DNA (cDNA) was reverse transcribed using a PrimeScript RT Reagent kit with gDNA Eraser (TaKaRa, RR047A) according to the manufacturer's instructions. The miR23b-5p primers were synthesized by RiboBio (Guangzhou, China). The qRT-PCR procedure used to detect the miR23b-5p level was: cycle $1,95^{\circ} \mathrm{C}$ for $2 \mathrm{~min}$; cycle 2 through 
$40,95^{\circ} \mathrm{C}$ for $30 \mathrm{~s}, 60^{\circ} \mathrm{C}$ for $35 \mathrm{~s}$, and fluorescence signal was detected at the end of each cycle. The expression level of the specific transcripts was normalized to internal controls ( $\beta$-actin or U6). The primers used in cyclin D1, myc and FOXM1 mRNA detection were shown as follows. Cyclin D1 forward: $5^{\prime}$-AACTACCTGGACCGCTTCCT$3^{\prime}$, reverse: 5'-CCACTTGAGCTTGTTCACCA-3'. MYC forward: $5^{\prime}$-TCAAGAGGCGAACACACAAC- ${ }^{\prime}$, reverse: 5'-GGCCTTTCATTGTTTTCCA-3'. FOXM1 forward: 5'CGTCGGCCACTGATTCTCAAA- $3^{\prime}$, reverse: $5^{\prime}$ - GGCA GGGGATCTCTTAGGTTC- $-3^{\prime}$. $\beta$-actin forward: $5^{\prime}$-TGG CACCCAG CACAATGAA-3', reverse: 5'-CTAAGTCA TAGTCCGCCTAGAAGCA- $3{ }^{\prime}$. Detection of each sample was repeated 3 times and the results were calculated using the $2^{-\Delta \Delta \mathrm{CT}}$ method.

\section{Establishment of stably transfected cells}

We purchased commercially available LV-hsa-miR-23b5p-mimic (pre-miR-23b-5p), LV-hsa-miR-23b-5p-inhibitor (miR-23b-5p-inhibitor), LV-has-miR-23b-5p-NC, LV-FOXM1, and LV-FOXM1-NC constructed in lentiviral vectors from GenePharma (Shanghai, China). After infecting the lentiviruses with HCC-LM3 and Hep3B cells, puromycin (Sigma-Aldrich, USA) was used to select cells according to protocols.

\section{Cell counting Kit-8 assay}

The effect of miR-23b-5p on cell growth was evaluated with a Cell Counting Kit-8 (CCK-8, Dojindo,Tokyo, Japan). Transfected cells were incubated in 96-well plates at a density of $1 \times 10^{3}$ cells per well and cultured as previously described.10ul of CCK-8 solution was mixed with $100 \mathrm{ul}$ serum-free medium and added to each well every $24 \mathrm{~h}$. After $2 \mathrm{~h}$ of incubation, we detected the absorbance at $450 \mathrm{~nm}$ in a microplate reader. The cells were cultured for 5 days.

\section{Colony formation assay}

We seeded 500 cells per well into 6-well plates and cultured as described before to evaluate the colony formation ability. We observed colonies every day and after the cells were cultured for 10 days. The medium was removed and washed by PBS for 3 times. The cells were fixed with ethyl alcohol for $30 \mathrm{~s}$ and stained with $1 \%$ crystal violet for $15 \mathrm{~min}$. The numbers of colonies were counted manually.

\section{5-ethynyl-2'-deoxyuridine (Edu) proliferation assay}

EdU proliferation assay (Ribobio, China) was used to evaluate the cell proliferation ability. Cells in the logarithmic growth phase were seeded in 24-well plates at 10,000 cells per well for 1 day. On the following day, cells were incubated with EdU for $2 \mathrm{~h}$. Then, cells were neutralized with $2 \mathrm{mg} / \mathrm{ml}$ glycine and permeabilized in $0.5 \%$
Triton X-100 for $20 \mathrm{~min}$. After extensive washing with PBS, cells were incubated with Apollo staining reaction buffer for $30 \mathrm{~min}$. Subsequently, the nuclei of cells were stained with DAPI for $15 \mathrm{~min}$, and the EdU incorporation rate was analyzed with a fluorescence microscope.

\section{Flow cytometric analysis}

To analyze cell cycle, sufficient amounts of cells were collected and added with $70 \%$ prechilled ethanol for $2 \mathrm{~h}$ overnight. On the next day, cells were resuspended in $500 \mu \mathrm{l}$ of PBS and then stained with $500 \mu \mathrm{l}$ propidium iodide (PI) (Vazyme, Nanjing, China) for $30 \mathrm{~min}$. The stained cells were analyzed by a BD FACSCanto II (BD Biosciences, USA) flow cytometer. The percentage of the cells in G1, S, and G2/M phase was analyzed using ModFit software.

\section{Western blot analysis}

Total protein was isolated from HCC tissues, cell lines and mouse tumors. BCA kit (Beyotime) was used to measure the protein concentration according to the manufacturer's instructions. The extracted protein was separated by a $10 \%$ SDS-PAGE based on their molecular weight and transferred to a polyvinylidene fluoride (PVDF) membrane (Bio-Rad, CA, USA). After blocking with $5 \%$ nonfat dry milk for $2 \mathrm{~h}$, the membranes were incubated with the appropriate primary antibody overnight at $4{ }^{\circ} \mathrm{C}$. Antibodies including rabbit anti-FOXM1 polyclonal antibody, anti-cyclinD1, anti-c-myc were used, and $\beta$-actin was used as an internal control. All antibody were purchased from Cell Signaling Technology, Danvers, MA, USA. Membranes were then incubated with HRPconjugated anti-rabbit IgG (1:2000) for $2 \mathrm{~h}$ at room temperature and then washed with TBST buffer three times. Protein expression levels were detected by ECL Plus (EMD Millipore, Billarica, MA, USA).

\section{Fluorescence in situ hybridization (FISH)}

We performed FISH to evaluate miR-23b-5p expression in HCC tissues and matched normal tissues. The mature human miR-23b-5p sequence is $3^{\prime}$ - UUUAGUCGUACGGUCCUUGGGU-5'. We purchased the miR-23b-5p antisense oligonucleotide probes from Servicebio (Wuhan, China). The FISH assay was conducted as previously described ${ }^{43}$.

\section{Immunohistochemical staining}

The HCC tissues were fixed in $4 \%$ paraformaldehyde, embedded in paraffin and cut into $4-\mu \mathrm{m}$ sections. After blocking endogenous peroxides and proteins, the sections were incubated with the primary antibody FOXM1and Ki67 (Cell Signaling Technology, Danvers, MA, USA). On the following day, the sections were incubated with secondary antibody at $37^{\circ} \mathrm{C}$ for $1 \mathrm{~h}$, incubated with 
3,3'-diaminobenzidine solution for $3 \mathrm{~min}$ and then counterstained with hematoxylin. The tumor sections were examined in a blinded manner.

\section{Dual-luciferase reporter assay}

The potential binding sites of miR-23b-5p in the FOXM1 3' UTR were predicted by TargetScan7.2 and miRWalk. The wild-type 3'-UTR sequences or the mutated sequences of FOXM1 were inserted into the pmir-GLO-promoter vector (Promega, Madison, USA). We seeded cells into a 24-well plate the day before transfection. Then, luciferase reporter plasmids were cotransfected with the miR-23b-5p mimic or negative control using Lipofectamine 3000 (Invitrogen). Luciferase activities were measured with the Dual Luciferase Reporter Assay System (Promega, USA).

\section{Bioinformatic analysis}

Clinical information about hepatocellular carcinoma (50 HCC tissues and paired 50 normal hepatic tissues) was obtained from TCGA database (The Cancer Genome Atlas, https://cancergenome.nih.gov). Differentially expressed miRNAs and mRNAs were filtered using Bioconductor $\mathrm{R}$ version 3.4 .1 (https://www.r-project.org/) and "edgeR" program package. The screening thresholds were $\mid \log _{2}$ (fold change) $\mid>1$ and adjusted $P$-value $<0.05$.

\section{Tumor xenograft in animals}

For tumor growth assay, ten nude mice (aged 4 weeks) were obtained from the Animal Model Institute of Nanjing University (Nanjing, China). Nude mice were randomly divided into 2 groups ( $n=5$ per group). HCCLM3-NC and HCC-LM3-pre-miR-23b-5p stable cells $(2 \times$ $10^{6}$ cells) in $100 \mu \mathrm{l}$ PBS were injected to nude mice by subcutaneous injection. We recorded the tumor size every 3 days and euthanized the mice 30 days later.

\section{Statistical analysis}

Data are expressed as mean \pm standard deviation from at least three experiments. The $\chi^{2}$ test was used to analyze the association of miR-23b-5p expression with clinicopathological features. Independent $t$-tests were used to compare two groups' differences. A paired $t$-test was used to analyze miR-23b-5p and FOXM1 mRNA levels in tissue samples. Correlations between miR-23b-5p and FOXM1 were determined by Pearson's correlation analysis. Statistical analyses were performed using GraphPad software and SPSS, ${ }^{*} P<0.05,{ }^{* *} P<0.01$, and ${ }^{* * *} P<0.001$ were defined as statistically significant.

\section{Acknowledgements}

We thank all those who participated in this study. This project was supported by the National Natural Science Foundation of China $(82070675,81871259$, 81530048, 81971495, and 81570562), the Foundation of Jiangsu Collaborative Innovation Center of Biomedical Functional Materials, the Priority Academic
Program Development of Jiangsu Higher Education Institutions, The Six talent peaks project in Jiangsu Province (2017-WSW-019).

Conflict of interest

The authors declare no competing interests.

\section{Publisher's note}

Springer Nature remains neutral with regard to jurisdictional claims in published maps and institutional affiliations.

Supplementary information The online version contains supplementary material available at https://doi.org/10.1038/s41420-021-00440-0.

Received: 12 December 2020 Revised: 29 January 2021 Accepted: 13 February 2021

Published online: 15 March 2021

\section{References}

1. Wei, W., Chua, M.-S., Grepper, S. \& So, S. Small molecule antagonists of Tcf4/ beta-catenin complex inhibit the growth of HCC cells in vitro and in vivo. Int. J Cancer 126, 2426-2436 (2010).

2. Siegel, R. L., Miller, K. D. \& Jemal, A. Cancer statistics, 2019.Cancer J. Clin. 69 , 7-34 (2019).

3. Greten, T. F., Wang, X. W. \& Korangy, F. Current concepts of immune based treatments for patients with HCC: from basic science to novel treatment approaches. Gut 64, 842-848 (2015).

4. Hanahan, D. \& Weinberg, R. A. Hallmarks of cancer: the next generation. Cell 144, 646-674 (2011).

5. Wang, L., Zou, Z. Q., Liu, C. X. \& Liu, X. Z. Immunotherapeutic interventions in chronic hepatitis B virus infection: a review. J. Immunol. Methods 407, 1-8 (2014).

6. Hayes, J., Peruzzi, P. P. \& Lawler, S. MicroRNAs in cancer: biomarkers, functions and therapy. Trends Mol. Med. 20, 460-469 (2014).

7. Boureima Oumarou, D. et al. Involvement of microRNA-23b-5p in the promotion of cardiac hypertrophy and dysfunction via the HMGB2 signaling pathway. Biomed. Pharmacother. 116, 108977 (2019).

8. You, L. et al. The role of microRNA-23b-5p in regulating brown adipogenesis and thermogenic program. Endocr. Connect 9, 457-470 (2020).

9. Hu, G.-D. et al. Long noncoding RNA CCAT2 functions as a competitive endogenous RNA to regulate FOXC1 expression by sponging miR-23b-5p in lung adenocarcinoma. J. Cell. Biochem. (2018). https://doi.org/10.1002/ jcb.28077

10. Shang, $X$. et al. Comprehensive circular RNA profiling reveals that hsa_circ_0005075, a new circular RNA biomarker, is involved in hepatocellular crcinoma development. Medicine 95, e3811 (2016).

11. Kalin, T. V., Ustiyan, V. \& Kalinichenko, V. V. Multiple faces of FoxM1 transcription factor: lessons from transgenic mouse models. Cell Cycle 10, 396-405 (2011).

12. Laoukili, J., Stahl, M. \& Medema, R. H. FoxM1: at the crossroads of ageing and cancer. Biochim. Biophys. Acta 1775, 92-102 (2007).

13. Gartel, A. L. A new target for proteasome inhibitors: FoxM1. Expert Opin. Investig. Drugs 19, 235-242 (2010).

14. Wang, $X$. et al. Increased levels of forkhead box M1B transcription factor in transgenic mouse hepatocytes prevent age-related proliferation defects in regenerating liver. Proc. Natl Acad. Sci. USA 98, 11468-11473 (2001).

15. Wierstra, I. \& Alves, J. Cyclin E/Cdk2, P/CAF, and E1A regulate the transactivation of the c-myc promoter by FOXM1. Biochem. Biophys. Res. Commun. 368, 107-115 (2008)

16. Zhang, Z., Zhang, G. \& Kong, C. FOXM1 participates in PLK1-regulated cell cycle progression in renal cell cancer cells. Oncol. Lett. 11, 2685-2691 (2016).

17. Li, Y., Zhang, S. \& Huang, S. FoxM1: a potential drug target for glioma. Future Oncol. 8, 223-226 (2012)

18. Kelleher, F. C. \& O'Sullivan, H. FOXM1 in sarcoma: role in cell cycle, pluripotency genes and stem cell pathways. Oncotarget 7, 42792-42804 (2016).

19. Gong, A. \& Huang, S. FoxM. 1 and Wnt/ $\beta$-catenin signaling in glioma stem cells. Cancer Res. 72, 5658-5662 (2012).

20. Senfter, D. et al. High impact of miRNA-4521 on FOXM1 expression in medulloblastoma. Cell Death Dis. 10, 696 (2019).

21. Wang, Y. et al. Dysregulation of miR-6868-5p/FOXM1 circuit contributes to colorectal cancer angiogenesis. J. Exp. Clin. Cancer Res. 37, 292 (2018). 
22. Zhi, Y. et al. FOXM1-mediated LINC-ROR regulates the proliferation and sensitivity to sorafenib in hepatocellular carcinoma. molecular therapy. Nucleic Acids Res. 16, 576-588 (2019).

23. Song, B.-N. \& Chu, I.-S. A gene expression signature of FOXM1 predicts the prognosis of hepatocellular carcinoma. Exp. Mol. Med. 50, e418 (2018).

24. Xia, L. et al. Upregulated FoxM1 expression induced by hepatitis B virus $X$ protein promotes tumor metastasis and indicates poor prognosis in hepatitis B virus-related hepatocellular carcinoma. J. Hepatol. 57, 600-612 (2012).

25. Callegari, E. et al. MicroRNAs in liver cancer: a model for investigating pathogenesis and novel therapeutic approaches. Cell Death Differ. 22, 46-57 (2015).

26. Farina, N. H. et al. A microRNA/Runx1/Runx2 network regulates prostate tumor progression from onset to adenocarcinoma in TRAMP mice. Oncotarget $\mathbf{7}$, 70462-70474 (2016).

27. Warnecke-Eberz, U., Chon, S.-H., Hölscher, A. H., Drebber, U. \& Bollschweiler, E. Exosomal onco-miRs from serum of patients with adenocarcinoma of the esophagus: comparison of miRNA profiles of exosomes and matching tumor. Tumour Biol. 36, 4643-4653 (2015).

28. Barrera-Ramirez, J. et al. Micro-RNA profiling of exosomes from marrowderived mesenchymal stromal cells in patients with acute myeloid leukemia: implications in leukemogenesis. Stem Cell Rev. Rep. 13, 817-825 (2017).

29. Nandi, D., Cheema, P. S., Jaiswal, N. \& Nag, A. FoxM1: repurposing an oncogene as a biomarker. Semin. Cancer Biol. 52, 74-84 (2018).

30. Laoukili, J. et al. FoxM1 is required for execution of the mitotic programme and chromosome stability. Nat. Cell Biol. 7, 126-136 (2005).

31. Tan, Y., Raychaudhuri, P. \& Costa, R. H. Chk2 mediates stabilization of the FoxM1 transcription factor to stimulate expression of DNA repair genes. Mol. Cell Biol. 27, 1007-1016 (2007).
32. Park, H. J. et al. FoxM1, a critical regulator of oxidative stress during oncogenesis. EMBO J. 28, 2908-2918 (2009).

33. Varghese, $\mathrm{V}$. et al. FOXM1 modulates 5-FU resistance in colorectal cancer through regulating TYMS expression. Sci. Rep. 9, 1505 (2019).

34. Xue, J. et al. Forkhead Box M1 is essential for nuclear localization of gliomaassociated oncogene homolog 1 in glioblastoma multiforme cells by promoting importin-7 expression. J. Biol. Chem. 290, 18662-18670 (2015).

35. Schüller, U. et al. Forkhead transcription factor FoxM1 regulates mitotic entry and prevents spindle defects in cerebellar granule neuron precursors. Mol. Cell Biol. 27, 8259-8270 (2007).

36. Anders, L. et al. A systematic screen for CDK4/6 substrates links FOXM1 phosphorylation to senescence suppression in cancer cells. Cancer Cell 20, 620-634 (2011).

37. Wang, I. C. et al. Foxm1 mediates cross talk between Kras/mitogen-activated protein kinase and canonical Wnt pathways during development of respiratory epithelium. Mol. Cell Biol. 32, 3838-3850 (2012).

38. Zhang, N. et al. FoxM1 promotes $\beta$-catenin nuclear localization and controls Wnt target-gene expression and glioma tumorigenesis. Cancer Cell $\mathbf{2 0}$ 427-442 (2011)

39. Dang, C. V. MYC on the path to cancer. Cell 149, 22-35 (2012).

40. Hunter, T. \& Pines, J. Cyclins and cancer. Cell 66, 1071-1074 (1991).

41. Motokura, T. \& Arnold, A. Cyclin D and oncogenesis. Curr. Opin. Genet. Dev. 3, 5-10 (1993).

42. Lu, J.W. et al. Clinical implications of deregulated CDK4 and Cyclin D1 expression in patients with human hepatocellular carcinoma. Med. Oncol. $\mathbf{3 0}$ 379 (2013).

43. Peng, F. et al. H19/let-7/LIN28 reciprocal negative regulatory circuit promotes breast cancer stem cell maintenance. Cell Death Dis. 8, e2569 (2017). 